\title{
Manipulating Focal Sets on the Unit Simplex: Application to Plastic Sorting
}

\author{
$1^{\text {st }}$ Lucie Jacquin \\ EuroMov Digital Health in Motion \\ Univ Montpellier, IMT Mines Ales \\ Ales, France \\ lucie.jacquin@mines-ales.fr \\ $4^{\text {th }}$ François Trousset \\ Laboratoire des Sciences des Risques (LSR) \\ IMT Mines Ales \\ Ales, France \\ francois.trousset@mines-ales.fr
}

\author{
$2^{\text {nd }}$ Abdelhak Imoussaten \\ EuroMov Digital Health in Motion \\ Univ Montpellier, IMT Mines Ales \\ Ales, France \\ abdelhak.imoussaten@mines-ales.fr
}

\author{
$3^{\text {rd }}$ Sebastien Destercke \\ Sorbonne universités, UTC \\ CNRS, Heudiasyc
}

57 Avenue de Landshut, Compiègne

sebastien.destercke@hds.utc.fr

$6^{\text {th }}$ Didier Perrin

$\begin{array}{cc}\text { EuroMov Digital Health in Motion } & \text { Polymers Composites and Hybrids }(\mathrm{PCH}) \\ \text { Univ Montpellier, IMT Mines Ales } & \text { IMT Mines Ales } \\ \text { Ales, France } & \text { Ales, France } \\ \text { jacky.montmain@mines-ales.fr } & \text { didier.perrin@mines-ales.fr }\end{array}$

\begin{abstract}
Belief functions are quite generic models when it comes to represent uncertain data, as it extends a wide range of uncertainty models (possiblity and probability distributions, among others). Usually, belief functions are defined over finite spaces, however many real word problems require to deal with beliefs over a continuous space while maintaining computational efficiency. This paper discusses the case of focal sets on the unit simplex, and proposes efficient inference tools to manipulate them. Such sets can be used to represent unknown proportions that one may face in various fields like soil contamination managing, plastic sorting or image reconstruction. In this paper, we illustrate their use on an industrial problem of plastic sorting, where the proportion of material impurities must not go over a limit while minimizing the rejection of sorted materials, whose nature is uncertain.
\end{abstract}

Index Terms-belief function, continuous focal sets, inference, plastic sorting.

\section{INTRODUCTION}

Belief functions [1] [2] are very rich uncertainty models, extending many existing models such as fuzzy sets and probability distributions [3]. Usually, belief functions are defined over finite spaces, however many problems require to maintain belief over a continuous space, even if it is based on a finite, discrete number of items of information. See, for example [4] that deals with the bivariate real space.

A specific case is the one where we are interested in focal sets (subsets receiving a positive mass assignment) that lives in the $n$-unit simplex, i.e., the set of positive vectors with $n$ elements $\theta_{1}, \ldots, \theta_{n}$ summing up to one. Indeed, such vectors can represent unknown proportions such as the composition of mixed components in a soil (to assess the amount of pollutants), in a container (to assess whether it is pure enough in one given component), or ill-known histograms (e.g., of pixels of a given colour in an image). Dealing with such information may be difficult in general, as focal elements are

Part of this work has been achieved during a visit of L. Jacquin to Heudiasyc, thanks to the CNRS AI research group (GDR IA) possibly infinite, and may take various forms. In practice, it is therefore necessary to simplify the problem and deal with specific subsets of the initial continuous space.

In this paper, we consider that we receive imprecise information about the vectors of proportions coming in the form of interval-valued proportions, but have precise information about their relative weights. More precisely, we will focus on the addition of such vectors. Section II describes our proposal, as well as some efficient ways to perform inferences. Section III illustrates this on a real-case scenario of plastic sorting where the goal is to recycle the plastic, and therefore to maintain the degree of impurity of a given container below a given level.

\section{FORMAL DESCRIPTION}

We consider that we have $n$ kinds $s_{1}, \ldots, s_{n}$ of materials, elements, etc. in a given space, for instance a container. We also assume we have information about their proportions $\theta_{1}, \ldots, \theta_{n}$, i.e., $\theta_{i} \in[0,1]$ and $\sum \theta_{i}=1$. We denote $\Theta=\left\{\left(\theta_{1}, \ldots, \theta_{n}\right) \in[0,1]^{n} \mid \sum_{s_{i}} \theta_{i}=1\right\}$ the set of all possible proportions.

\section{A. Addition of imprecise proportion vectors}

In this section, we consider that new information about the proportions will come as a precisely known total weight of new elements with imprecise information about their possible proportions. More particularly, an imprecise piece of information $j$ will be formed of two components:

- a precise weight $w^{j} \in \mathbb{R}_{+}^{*}$ of elements counted up to now (in number of pixels, of soil area, of mass in the container, ... )

- intervals $\mathcal{I}^{j}=\left\{\left[\ell^{j}, u^{j}\right]\right\}$, where $\left[\ell_{i}^{j}, u_{i}^{j}\right] \subseteq[0,1]$ denotes our imprecise knowledge about the proportions $\theta_{i}$ of materials, elements that are of the kind $s_{i}$. As these intervals must describe imprecisely known proportions, they should contain at least one precise set $\theta_{1}, \ldots, \theta_{n}$ such that $\theta_{i} \in\left[l_{i}^{j}, u_{i}^{j}\right]$ i.e., $\sum_{i} \ell_{i}^{j} \leq 1 \leq \sum_{i} u_{i}^{j}[5]$, and be 
reachable, meaning that there exists at least one vector of proportions $\left(\theta_{1}, \ldots, \theta_{n}\right)$ with either $\theta_{i}=l_{i}^{j}$ or $\theta_{i}=u_{i}^{j}$, and this for every $i \in\{1, \ldots, n\}$, i.e., $\sum_{j \neq i} l_{j}+u_{i} \leq 1$ and $\sum_{j \neq i} u_{j}+l_{i} \geq 1, \forall i$ [5]. Note that here, we will automatically satisfy such requirements, as bounds come from physical observations.

We now consider that we have an initial information $w^{1}, \mathcal{I}^{1}=$ $\left\{\left[\ell^{1}, u^{1}\right]\right\}$ representing the current total weights of items and their partially known proportions, and that we want to add a new item of imprecise information $w^{2}, \mathcal{I}^{2}=\left\{\left[\ell^{2}, u^{2}\right]\right\}$. The weight of their combination will be $w^{1}+w^{2}$, and the combined intervals will be denoted by $\mathcal{I}^{1} \boxplus \mathcal{I}^{2}$ where

$$
\left[\ell_{i}^{1}, u_{i}^{1}\right] \boxplus\left[\ell_{i}^{2}, u_{i}^{2}\right]=[l, u]
$$

with

$$
l=\frac{w^{1}}{w^{1}+w^{2}} \ell_{i}^{1}+\frac{w^{2}}{w^{1}+w^{2}} \ell_{i}^{2},
$$

and

$$
u=\frac{w^{1}}{w^{1}+w^{2}} u_{i}^{1}+\frac{w^{2}}{w^{1}+w^{2}} u_{i}^{2} .
$$

Example 1: Consider that we have three kinds of materials $s_{1}, s_{2}, s_{3}$ and we have two pieces of information. The first information concerns a container with total weight $w^{1}=1 \mathrm{~kg}$ of different objects containing a proportion $\theta_{1} \in[1 / 4,1 / 2]$ of $s_{1}$, a proportion $\theta_{2} \in[0,1 / 3]$ of $s_{2}$ and a proportion $\theta_{3} \in$ $[1 / 6,3 / 4]$ of $s_{3}$. The second piece of information concerns a new element added to the container with the same weight $w^{2}=$ $1 \mathrm{~kg}$ and with proportions $\theta_{1} \in[0,1 / 2], \theta_{2} \in[0,1 / 2], \theta_{3} \in$ $[0,1 / 2]$ (no material exceeds half of the total weight). When the new piece is added to the container, we will have the following proportions:

$$
\mathcal{I}^{1} \boxplus \mathcal{I}^{2}=\left(\left[\frac{1}{8}, \frac{1}{2}\right],\left[0, \frac{5}{12}\right],\left[\frac{1}{12}, \frac{5}{8}\right]\right)
$$

Remark 1: A particular type of vectors often found in applications, and in particular plastic sorting, is the case where it is assumed that the new element is pure, i.e., is made of only one kind among $s_{1}, \ldots, s_{n}$, but where we only know that this element belongs to a subset $\emptyset \neq E \subseteq\left\{s_{1}, \ldots, s_{n}\right\}$. In this case, the convex closure of the corresponding information item $\mathcal{I}_{i}^{j}$ is

$$
\mathcal{I}_{i}^{j}=\left\{\begin{array}{l}
\{0\} \text { if } s_{i} \notin E, \\
\{1\} \text { if } E=\left\{s_{i}\right\}, \\
{[0,1] \text { if } s_{i} \in E \text { and }|E|>1 .}
\end{array}\right.
$$

Without considering the convex hull, the last item should be $\{0,1\}$, yet as all subsequent inferences will be linear, and therefore will be obtained on extreme points, considering the hull will not change them, and will considerably simplify our exposure.

\section{B. From imprecise proportions to evidential proportions}

In practice, we may have more refined information about the proportions than a purely imprecise information. Belief functions and mass assignments are very flexible models of uncertainty that encompass in one sweep intervals, possibility distributions (a.k.a. fuzzy sets describing uncertainty), probability masses, etc.. In particular, we will consider in our application (Section III) that information about the elements added to a container is given by a classifier outputting belief functions.

A belief function on a space $\Theta$ (here the space of all proportion vectors) is formally equivalent to a mass assignment $m: \mathcal{P}(\Theta) \rightarrow[0,1]$ where $\mathcal{P}(\Theta)$ is the power set of $\Theta$. From such a mass can then be defined different measures, such as the belief and plausibilities in an event $A \subseteq \Theta$, defined as

$$
\begin{gathered}
\operatorname{Bel}(A)=\sum_{E \subseteq \Theta, E \subseteq A} m(E) \\
P l(A)=\sum_{E \subseteq \Theta, E \cap A \neq \emptyset} m(E) .
\end{gathered}
$$

These measures are dual, in the sense that $\operatorname{Bel}(A)=1-$ $\operatorname{Pl}\left(A^{c}\right)$. Here, we will only consider subsets in the form of bounded proportion vectors $\mathcal{I}$. For a mass function $m^{j}$, we will denote by $\mathcal{F}^{j}$ the set of bounded proportion vectors having a positive mass.

Given two belief functions $m^{1}, m^{2}$ bearing on proportion vectors, associated to weights $w^{1}$ and $w^{2}$ of elements, we define the mass resulting from the sum $m^{1 \oplus 2}$ as

$$
m^{1 \oplus 2}(\mathcal{I})=\sum_{\substack{\mathcal{I}^{\prime} \in \mathcal{F}^{1}, \mathcal{I}^{\prime \prime} \in \mathcal{F}^{2} \\ \mathcal{I}=\mathcal{I}^{\prime} \boxplus \mathcal{I}^{\prime \prime}}} m^{1}\left(\mathcal{I}^{\prime}\right) \cdot m^{2}\left(\mathcal{I}^{\prime \prime}\right) .
$$

where $\boxplus$ is defined in formula (1) and the total weight associated to the mass function $m^{1 \oplus 2}$ is $w^{1}+w^{2}$. This operation can of course be repeated iteratively if we have more than one mass function. It should be noticed that if all mass functions have a finite number of focal elements, then so has $m^{1 \oplus 2}$, with the number being at most $\left|\mathcal{F}^{1}\right| \cdot\left|\mathcal{F}^{2}\right|$. In practice we may have to approximate the results to control the number of focal elements if this number becomes too big.

Example 2: Let us extend Example 1, Subsection II-A where evidential information is considered instead of imprecise information. TABLE I gives the two masses concerning the container $m^{1}$ and the new object $m^{2}$.

TABLE I

THE TWO PIECES OF EVIDENTIAL INFORMATION

\begin{tabular}{|cc|cc|}
\hline \multicolumn{2}{|c|}{ The container $\left(w^{1}=1 \mathrm{~kg}\right)$} & \multicolumn{2}{c|}{ The new object $\left(w^{1}=1 \mathrm{~kg}\right)$} \\
\hline $\mathcal{F}^{1}$ & $m^{1}$ & $\mathcal{F}^{2}$ & $\mathrm{~m}^{2}$ \\
\hline $\mathcal{I}_{1}^{\prime}$ & 0.5 & $\mathcal{I}_{1}^{\prime \prime}$ & 0.6 \\
{$[1 / 4,1 / 2] \times[0,1 / 3] \times[1 / 6,3 / 4]$} & & {$[0,1 / 2] \times[0,1 / 2] \times[0,1 / 2]$} & \\
$\mathcal{I}_{2}^{\prime}$ & 0.5 & $\mathcal{I}_{2}^{\prime \prime}$ & 0.4 \\
{$[0,1 / 6] \times[0,1 / 6] \times[5 / 6,1]$} & & {$[0,1 / 6] \times[0,1 / 6] \times[5 / 6,1]$} & \\
\hline
\end{tabular}


When the new piece is added to the container, we will have the following focal elements for $m^{1 \oplus 2}$ :

$$
\begin{aligned}
& \mathcal{I}_{1}^{\prime} \boxplus \mathcal{I}_{1}^{\prime \prime}=([1 / 8,1 / 2] \times[0,5 / 12] \times[1 / 12,5 / 8]), \\
& m^{1 \oplus 2}\left(\mathcal{I}_{1}^{\prime} \boxplus \mathcal{I}_{1}^{\prime \prime}\right)=0.3, \\
& \mathcal{I}_{1}^{\prime} \boxplus \mathcal{I}_{2}^{\prime \prime}=([1 / 8,1 / 3] \times[0,1 / 4] \times[1 / 2,7 / 8]), \\
& m^{1 \oplus 2}\left(\mathcal{I}_{1}^{\prime} \boxplus \mathcal{I}_{2}^{\prime \prime}\right)=0.2, \\
& \mathcal{I}_{2}^{\prime} \boxplus \mathcal{I}_{1}^{\prime \prime}=([0,1 / 3] \times[0,1 / 3] \times[5 / 12,3 / 4]), \\
& m^{1 \oplus 2}\left(\mathcal{I}_{2}^{\prime} \boxplus \mathcal{I}_{1}^{\prime \prime}\right)=0.3, \\
& \mathcal{I}_{2}^{\prime} \boxplus \mathcal{I}_{2}^{\prime \prime}=([0,1 / 6] \times[0,1 / 6] \times[5 / 6,1]), \\
& m^{1 \oplus 2}\left(\mathcal{I}_{2}^{\prime} \boxplus \mathcal{I}_{2}^{\prime \prime}\right)=0.2 .
\end{aligned}
$$

\section{Some inferences over evidential proportions}

Of course, once we have computed our uncertain knowledge about the element proportions, we will have to make some inferences about them, and in particular compute plausibilities/belief for specific events $A$. A particular kind of event (and its complement) we will be interested in are events of the kind "the $\%$ of materials of kind $A$ is below some threshold $\alpha$ ", where $A \subseteq\left\{s_{1}, \ldots, s_{n}\right\}$ is a subset of specific material kinds. One may think for instance of having a limited quantity of pollutants in the soil or a limited number of impurities in a container of plastic to recycle. Such kind of events, that we will denote by $A_{\alpha}$, simply translates into $\sum_{s_{i} \in A} \theta_{i} \leq \alpha$. More precisely, $A_{\alpha}$ is a subset of $\Theta$ such that:

$$
A_{\alpha}=\left\{\left(\theta_{1}, \ldots, \theta_{n}\right) \in[0,1]^{n}: \sum_{i=1}^{n} \theta_{i}=1 \text { and } \sum_{s_{i} \in A} \theta_{i} \leq \alpha\right\}
$$

Let us now consider a belief function $m$ defined over vector proportions. To compute $\left[\operatorname{Bel}\left(A_{\alpha}\right), \operatorname{Pl}\left(A_{\alpha}\right)\right]$, we will have to check whether focal elements are included, overlap or are disjoint with $A_{\alpha}$. For a given focal element $\mathcal{I} \in \mathcal{F}$, this can be done easily:

- We will have $\mathcal{I} \subseteq A_{\alpha}$ if the sums of all possible proportions in $\mathcal{I}$ of materials within $A$ are below the threshold $\alpha$. This can be done simply, as we have

$$
\mathcal{I} \subseteq A_{\alpha} \Leftrightarrow \min \left(\sum_{s_{i} \in A} u_{i}, 1-\sum_{s_{i} \notin A} \ell_{i},\right) \leq \alpha
$$

with the first term of the last inequality being the maximal proportion of material that can be given to $A$, given our imprecise knowledge $\mathcal{I}$.

- We will have $\mathcal{I} \cap A_{\alpha} \neq \emptyset$ if there is at least a proportion vector for which the proportion of materials given to $A$ is below $\alpha$. This can again be simply checked as we have

$$
\mathcal{I} \cap A_{\alpha} \neq \emptyset \Leftrightarrow \max \left(\sum_{s_{i} \in A} \ell_{i}, 1-\sum_{s_{i} \notin A} u_{i}\right) \leq \alpha
$$

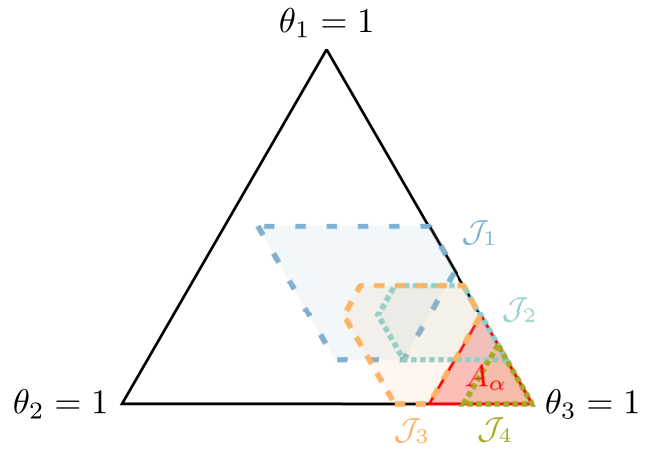

Fig. 1. Representation of the different subsets in the simplex

with the first term of the last inequality being the minimal proportion of material that can be given to $A$, given our imprecise knowledge $\mathcal{I}$. Clearly, if this proportion is below $\alpha$, then it proves that at least one vector is within $A_{\alpha}$, and if it is above, then no vectors will have a lower proportion in material within $A$, and therefore $\mathcal{I} \cap A_{\alpha}=\emptyset$.

Note that, in practice, such events can easily be combined. Assume for example that elements in $A$ should not be over a percentage $\alpha$, and that elements in $B$ should not be over a percentage $\beta$, then checking whether $\mathcal{I} \subseteq A_{\alpha} \cap B_{\beta}$ and $\mathcal{I} \cap\left(A_{\alpha} \cap B_{\beta}\right) \neq \emptyset$ simply amounts to perform the checks (8) and (7) for $A_{\alpha}$ and $B_{\beta}$ separately, as we are concerned by their intersection (this would not be true for their union). Also note that checking whether elements in a subset $C$ are above some threshold $\gamma$ simply comes down to check event $C_{1-\gamma}^{c}$. This remark of course extends to any number of events of this kind. In particular, one could check whether the percentage of a particular damaging material $\left\{s_{i}\right\}$ or pollutant is not too high (or if the amount of an interesting or necessary element is high enough).

To compute $\left[\operatorname{Bel}\left(A_{\alpha}\right), \operatorname{Pl}\left(A_{\alpha}\right)\right]$ and therefore $\left[\operatorname{Bel}\left(A_{\alpha}^{c}\right), P l\left(A_{\alpha}^{c}\right)\right]$, we only need to perform $|\mathcal{F}|$ the checks described above, or even less if $\mathcal{F}$ has a specific structure (e.g., nestedness of focal elements).

Example 3: Let us go back to Example 2, Subsection II-B. Let us consider $A=\left\{s_{1}, s_{2}\right\}$ and $\alpha=0.25$ (there should be at most $25 \%$ of type 1 and 2 , or equivalently at least $75 \%$ of type $3)$, TABLE II gives the results of different checks described above related to $A_{\alpha}$ and Fig. 1 shows the representation of the imprecise vectors in the unit simplex.

TABLE II

Computation of $\operatorname{Bel}\left(A_{\alpha}\right)$ and $\operatorname{Pl}\left(A_{\alpha}\right)$

\begin{tabular}{|cc|c|c|}
\hline & & Belief $\mathrm{Bel}$ & Plausibility $P l$ \\
\hline $\mathcal{J}$ & $m^{1 \oplus 2}(\mathcal{J})$ & $\mathcal{J} \subseteq A_{\alpha}$ & $\mathcal{J} \cap A_{\alpha} \neq \emptyset$ \\
\hline $\mathcal{J}_{1}=\mathcal{I}_{1}^{\prime} \boxplus \mathcal{I}_{1}^{\prime \prime}$ & 0.3 & no & no \\
$\mathcal{J}_{2}=\mathcal{I}_{1}^{\prime} \boxplus \mathcal{I}_{2}^{\prime \prime}$ & 0.2 & no & yes \\
$\mathcal{J}_{3}=\mathcal{I}_{2}^{\prime} \boxplus \mathcal{I}_{1}^{\prime \prime}$ & 0.3 & no & yes \\
$\mathcal{J}_{4}=\mathcal{I}_{2}^{\prime} \boxplus \mathcal{I}_{2}^{\prime \prime}$ & 0.2 & yes & yes \\
& & $\operatorname{Bel}\left(A_{\alpha}\right)=0.2$ & $\operatorname{Pl}\left(A_{\alpha}\right)=0.7$ \\
\hline
\end{tabular}




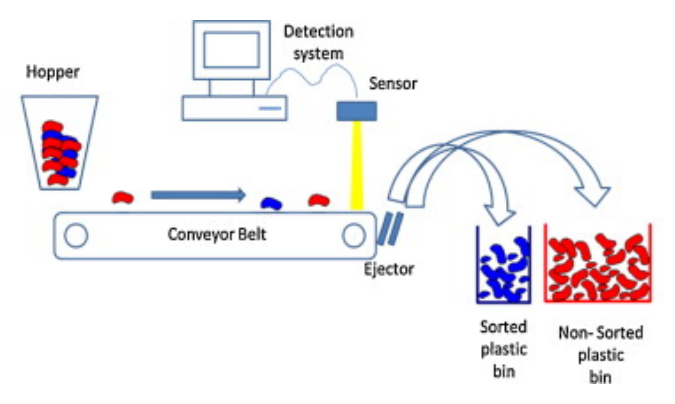

Fig. 2. Example of sorting device

\section{Application to Plastic SORTING}

In this section we present an application concerning the plastic sorting for recycling purposes in industrial conditions. Due to some physico-chemical reasons related to nonmiscibility, plastics must be sorted by types of materials prior to recycling. For some types of different materials that share close visual characteristics, manual sorting is infeasible. Moreover the throughput of sorting is demanding. Optical sorting devices provide benefits for these issues. Fig. 2, borrowed from [6] illustrates such device. In practice, fragments of plastics arrive continuously on a conveyor belt before being recorded by an infra-red camera. The information acquired for each fragments is then used to decide the sorting of the fragments, i.e., to which container the fragments should be sent. However, the acquired information is subject to several issues inducing the presence of imprecision, i.e. some features information are not precise enough to draw clear distinctions between the materials type, and uncertainty, i.e. some obtained features are not completely consistent with the reality. We refer to [7] for details. Two steps are considered in the sorting of a fragment. The first step consists to apply a classifier that is integrated to the sorting device. Then the second step is dedicated to perform on line sorting decision with constraints on the composition of the containers.

Since small quantity of badly sorted plastics can lead to high decreases of impact resistance [8] and of monetary value, impurities should by limited. Thus, experts have defined tolerance threshold on the proportions of impurities. The second step is used to check compliance with such constraints. In this application we focus only on the second step which is an illustration to the work presented in this paper. We consider therefore that the results of the first step are available and used as inputs for the second step.

\section{A. Data presentation}

Let us consider a plastic waste deposit of three material types $s_{1}, s_{2}, s_{3}$, we denote their set by $S=\left\{s_{1}, s_{2}, s_{3}\right\}$. Let us consider a fragment $f$ to be treated by the sorting device. The fragment $f$ is composed of the proportion $\theta_{1}$ of $s_{1}$, the proportion $\theta_{2}$ of $s_{2}$ and the proportion $\theta_{3}$ of $s_{3}$. To simplify we consider only one container that is dedicated to the plastics respecting some constraints and all other fragments are rejected. The sorting devise has two options for the fragment $f$
1) blowing it to the container or 2) rejecting it. The procedure could be easily generalised to more possible options by adding other containers.

For example, we can consider that the proportions of materials of kinds $A \subseteq S$ should not exceed some thresholds $\alpha$ in the container. We use the set $A_{\alpha}$ defined in (6), to express this kind of constraints. This means that this container is dedicated for $A^{c}$ and elements of $A$ are impurities. We refer to "positive sorting" when the fragment is blown to the container.

As mentioned before the information about the vector of proportions $\left(\theta_{1}, \theta_{2}, \theta_{3}\right)$ related to $f$ is provided by a classifier. More precisely, we consider the case of a multi-label classifier which provide us with the set of the material types present in the fragment $f$. The information provided is a posterior probability $p(. \mid f)$ of the presence of a combination of elements of $S$ in the fragment $f$. In the following we adopt the notation $s_{i} \wedge s_{j}$ to say that the fragment is composed of material types $s_{i}$ and $s_{j}$. To simplify we consider only the cases where the classifier hesitates between two compositions, e.g., if the classifier hesitates between two compositions $s_{1}$ and $s_{1} \wedge s_{3}$, we have $p\left(s_{1} \mid f\right)+p\left(s_{1} \wedge s_{3} \mid f\right)=1$.

In the latter example where the classifier hesitates to consider $f$ as a pure material type $s_{1}$ or as a combination of both material types $s_{1}$ and $s_{3}$, let us consider that it provides the following probabilities: $p\left(s_{1} \mid f\right)=0.4$, and $p\left(s_{1} \wedge s_{3} \mid f\right)=0.6$. Note that the information " $p\left(s_{1} \wedge s_{3} \mid f\right)=0.6$ " does not inform us about the proportions of materials $s_{1}$ and $s_{3}$ in $f$ but only about their possible presence in the fragment, i.e., we only know that the proportions $\theta_{1}$ and $\theta_{3}$ are in $] 0,1[$, that we will transform into the closed interval $[0,1]$ without loss of relevant information in our case (see Remark 1). Finally, from this information about the presence of materials in fragment $f$ one can express a mass function $m(. \mid f)$ on the proportions of these materials. The corresponding focal elements $\mathcal{I}^{s_{1}}$ and $\mathcal{I}^{s_{1} \wedge s_{3}}$ are:

$$
\begin{gathered}
\mathcal{I}^{s_{1}}=([1,1],[0,0],[0,0]), \\
\mathcal{I}^{s_{1} \wedge s_{3}}=([0,1],[0,0],[0,1]),
\end{gathered}
$$

where $\mathcal{I}^{s_{1}}$ is an information coming from the output of the classifier: $p\left(s_{1} \mid x\right)=0.4$ and $\mathcal{I}^{s_{1} \wedge s_{3}}$ from $p\left(s_{1} \wedge s_{3} \mid x\right)=0.6$. The probability masses are transferred to $\mathcal{I}^{s_{1}}$ and $\mathcal{I}^{s_{1} \wedge s_{3}}$ :

$$
m\left(\mathcal{I}^{s_{1}} \mid f\right)=0.4, \quad m\left(\mathcal{I}^{s_{1} \wedge s_{3}} \mid f\right)=0.6
$$

More generally, let us consider a fragment $f$ for which the classifier provides a non-null probability for the composition $C$ of materials. The corresponding imprecise vector of proportions is then $\mathcal{I}^{C}=\left(\left[l_{1}, u_{1}\right],\left[l_{2}, u_{2}\right],\left[l_{3}, u_{3}\right]\right)$ where:

$$
\left[l_{i}, u_{i}\right]=\left\{\begin{array}{l}
{[0,0] \text { if } s_{i} \notin C,} \\
{[1,1] \text { if } C=s_{i},} \\
{[0,1] \text { if } s_{i} \in C \text { and }|C|>1}
\end{array}\right.
$$

Furthermore, weighing precisely each small fragments is a fastidious task, consequently we consider in this application that the weight of a fragment is approximated by the number of pixels in its acquired image. 


\section{B. Updating bucket composition with classifiers}

Consider that $f$ is the new fragment to treat by the sorting device, we have the following information about $f$ :

- its weight $W^{f}$,

- its mass function $m(. \mid f)$,

Concerning the container that contains $k$ fragments, we have the following information before treating the fragment $f$ :

- the current weight $W_{k}^{\text {cont }}$ of the fragments it contains,

- the mass function $m_{k}$ describing our knowledge of the current proportions of materials it contains.

If $f$ is added to the container, the current weight $W_{k}^{\text {cont }}$ and the mass function $m_{k}$ are updated using the sum operation (5):

$$
\left(m_{k+1}, W_{k+1}^{c o n t}\right)=\left(m_{k} \oplus m(. \mid f), W_{k}^{c o n t}+W^{f}\right)
$$

From $m_{k+1}$, we can estimate our belief of whether the impurities proportions is not currently too high by computing $\operatorname{Bel}_{k+1}\left(A_{\alpha}\right)$.

We denote $m_{\text {final }}$ the resulting mass function for the proportions in the container at the final step and $W$ the total capacity of the container, i.e., its weight at the end of the sorting.

\section{Making inference about bucket potential}

We think that the behaviour of the decision-maker should be designed carefully, as it has a real impact on the final positive sorting rate, i.e., the amount of positively sorted material over the total amount of sorted materials when $W$ is reached . The higher this rate is, the faster the container is filled and thus the less material are rejected. However, while the container should be filled as fast as possible, it should also respect the impurity constraints.

So far we are able to track the evolution of our knowledge about the container's composition in real time and make inference about impurity constraints satisfaction. One could then base the sorting decision on the inference about the event $A_{\alpha}$. For instance, a decision procedure for the sorting of $f$ may rely on the potential resulting composition of the container if we add $f$ to it. Thus the sorting device would blow the fragment to the container only if:

$$
\operatorname{Bel}_{k} \oplus \operatorname{Bel}\left(A_{\alpha} \mid f\right) \geq \eta,
$$

with $\eta$ being a confidence threshold belonging in $[0,1]$ that should be large enough to limit the risk of having a bad container. Considering this decision for each successive fragment will ensure that at any time during the container filling, the belief that impurity constraints are respected will be higher than $\eta$. This is a very strong constraint, as only very pure elements added in the future could make $\operatorname{Bel}\left(A_{\alpha}\right)$ increase.

A more flexible procedure could be considered where the sorting device may blow some fragments to the container even if the resulting composition in the container leads to $\operatorname{Bel}_{k}\left(A_{\alpha}\right)<\eta$. Indeed, the constraints on the impurities are required to be satisfied only at the end of the filling of the container, i.e., when the amount of material in the container reaches the total weight $W$. The objective then is to verify

$$
\operatorname{Bel}_{\text {final }}\left(A_{\alpha}\right) \geq \eta \text {. }
$$

The idea behind the flexible procedure is to make hypotheses about the future coming fragments in order to better assess the benefit of adding the current fragment. Thus $\operatorname{Bel}_{\text {final }}\left(A_{\alpha}\right)$ needs to be estimated at any time. We think that a reasonable tolerance on the quality of fragments is preferable at the beginning, and more exigency is required at the end to compensate for the first more hazardous decisions. This leads us to consider rather optimistic thoughts about the future fragments. A simple hypothesis is to consider that in the short term, future fragments would be of the same nature of the current fragment and in the long term the last fragments will be "good" fragments, i.e., we would have some evidence that they are composed by materials of kinds $A^{c}$.

Let us consider $W_{\text {rest }}=W-W_{k}^{\text {cont }}$ the remaining weight required to fill up the container. We consider that the first half of the future filling fragments correspond to a first fictitious fragment $f^{\text {part } 1}$ of weight $W_{\text {rest }} / 2$ having a mass function $m^{\text {part } 1}$ identical to the mass function of the coming fragment, i.e., $m^{\text {part } 1}=m(. \mid f)$. On the other hand, we consider a second fictitious fragment $f^{\text {part2 }}$ of weigh $W_{\text {rest }} / 2$ for the second part of the container yet to fill. Traducing optimism on this second part, we chose to represent the belief about $f^{\text {part } 2}$ by a mass function that assigns a large mass for the event that $f^{\text {part2 } 2}$ is of kind $A^{c}$, i.e. the elements for which the container is dedicated. To obtain such mass function we can consider the focal element $\mathcal{I}^{\text {part } 2 \text { : }}$

$$
\mathcal{I}_{i}^{\text {part } 2}=\left\{\begin{array}{l}
{[0,0] \text { if } s_{i} \notin A^{c},} \\
{[1,1] \text { if } A^{c}=\left\{s_{i}\right\},} \\
{[0,1] \text { if } s_{i} \in A^{c} \text { and }\left|A^{c}\right|>1,}
\end{array}\right.
$$

then we define the mass function on the proportion of $f^{\text {part } 2}$ by:

$$
\begin{aligned}
m^{\text {part } 2}\left(\mathcal{I}^{\text {part } 2}\right) & =\gamma, \\
m^{\text {part } 2}(\Theta) & =1-\gamma,
\end{aligned}
$$

$\gamma \in[0,1]$, chosen according to our degree of optimism. See Fig. 3 for a recap of all information and hypotheses used for estimating the evidence about the final composition of the container. By considering the latter fictitious fragments, we can allow small risk taking at the beginning of the sorting, as a high $\gamma$ means that we hope future fragment can compensate previous risky decisions. Indeed, at the beginning $W_{\text {rest }}$ is high so our optimistic hypothesis has an important role in the evaluation of (11). However, as the sorting progress go on, the portion of future fragments will decrease, and so will its influence in the estimation of belief of the final composition. On the other hand the current state of the container composition will have an increasing influence in the evaluation of (11). Therefore the sorting would be more exigent when progressing to the final steps. 


\begin{tabular}{|c|c|c|}
\hline$m^{\text {part } 2}$ & $W_{\text {rest }} / 2$ & Long term future \\
\hline$m^{\text {part } 1}$ & $W_{\text {rest }} / 2$ & Short term future \\
\hline$m(. \mid f)$ & $W^{f}$ & Present, current fragment \\
\hline$m_{k}$ & $W_{k}^{\text {container }}$ & Past \\
\hline
\end{tabular}

Fig. 3. Estimation of the container composition under our simple hypothesis

TABLE III

AVERAGE VALUES OF $\operatorname{Bel}_{\text {final }}\left(A_{\alpha}\right)$ AND final positive sorting rate ON 15 SIMULATIONS

\begin{tabular}{c|c|c|c}
\hline & Naive & Cautious & Flexible \\
\hline Bel $_{\text {final }}\left(A_{\alpha}\right)$ & 0.666 & 0.613 & $\mathbf{0 . 6 7 8}$ \\
\hline Final positive sorting rate & $\mathbf{0 . 1 3 2}$ & 0.022 & 0.110 \\
\hline
\end{tabular}

\section{Simulations}

In this subsection we present simulations of the sorting scenario where the proportions of the plastic of material types $s_{1}$ and $s_{2}$ must be minimized in the container that is dedicated to material type $s_{3}$. To simplify the presentation, we assume that all fragments are composed of a single pixel, i.e., $w=10 \mathrm{~g}$. For each simulated fragment $f_{k}$, the probability $p_{k}$ is randomly generated (in practice, it would come from a classifier). Thus we used the following setting: $\eta=0.6$, $\alpha=0.25$ and $W=100 \mathrm{~g}$. We have considered different procedures for filling the container to sort a fragment $f$ :

- Naive procedure: the decision is only based on the information concerning the current fragment. If $\operatorname{Bel}\left(A_{\alpha}\right) \geq \eta$ is verified for $m(. \mid f)$ then fragment $f$ is positively sorted.

- Cautious procedure: the decision is based on the information concerning the current fragment $m(. \mid f)$ and the current composition of the container, i.e., $m_{k-1}$. If (10) is verified then fragment $f$ is positively sorted.

- Flexible procedure: as described in the previous subsection, this procedure is based on some estimation of the evidence on the container final composition. Eventually the procedure corresponds to sort positively $f$ only if our estimation of $m_{\text {final }}$ as $m_{k} \oplus m(. \mid f) \oplus m^{\text {part } 1} \oplus m^{\text {part } 2}$ implies that (11) is verified. In the simulations, we choose $m^{\text {part } 2}$ by setting $\gamma=0.8$ higher than $\eta$ traducing our degree of optimism.

The evolution of $\operatorname{Bel}_{k}\left(A_{\alpha}\right)$ is plotted regarding the sorting progress in Fig. 4, 5 and 6, respectively for the latter three approaches. Each curves represents a simulation and stops with a bullet marking the end of the sorting, i.e., filling of the container. Note that each simulation has different duration because of the rate of positive sorting, i.e., the higher the rejected fragment rate, the longer the duration. To better compare the results of the approaches, we used the same set seed simulations for the three procedures. The average on all simulation for $\operatorname{Bel}_{\text {final }}\left(A_{\alpha}\right)$ and for the final positive sorting rate are presented in TABLE III.
1) Naive procedure: This procedure has the advantage to quickly fill the container (see TABLE III). Yet as we can observe in Fig. 4 this procedure does not guarantee that (11) will be respected. In fact we could verify (11), by requiring much more exigency concerning each fragment $f_{k}$ added in the container, however doing so would not be feasible for industrial sorting where container capacity $W$ is large.

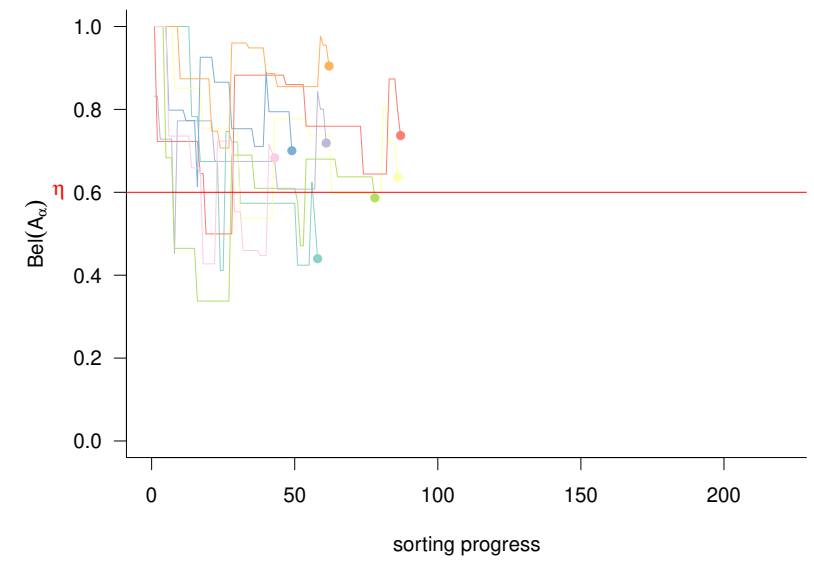

Fig. 4. Naive procedure

2) Cautious procedure: As mentioned in subsection III-C, this procedure imposes that for all step of the container filling, $k, \operatorname{Bel}_{k}\left(A_{\alpha}\right) \geq \eta$. The drawback of this procedure is that it only focuses on the current container's composition without anticipating the future. Hence, in favourable situation, i.e., $\operatorname{Bel}_{k}\left(A_{\alpha}\right)$ is much higher than $\eta$, some bad fragments could be added to the container without much harm. However, doing so successively and too often would decrease the composition's quality up to an unacceptable mix. Also, as we can see in Fig. 5, for some simulations the device sorting takes a very long time to fill the container (arrows are added to Fig. 5 to represent sorting simulations that end after more than 220 iterations).

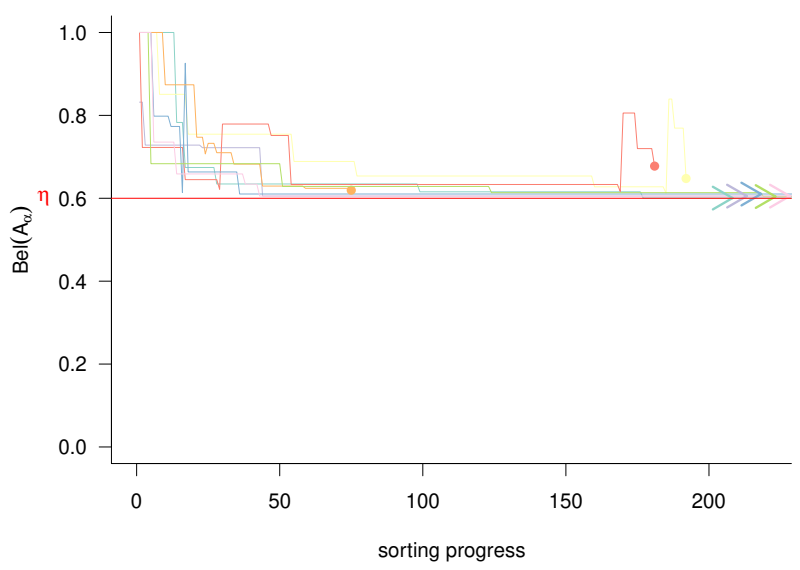

Fig. 5. Cautious procedure

3) Flexible procedure: In the flexible procedure we can have $\operatorname{Bel}_{k}\left(A_{\alpha}\right)<\eta$ during the sorting, but as we can see in 
(6), the restriction on impurities are always fulfilled in the end of the sorting. Moreover the rate of positive sorting at the end of the sorting is better than for the cautious procedure (see TABLE III). This is due to the fragments selection which is more suitable for the twofold objective, i.e., respecting (11) and minimizing the rejected fragments, in this procedure.

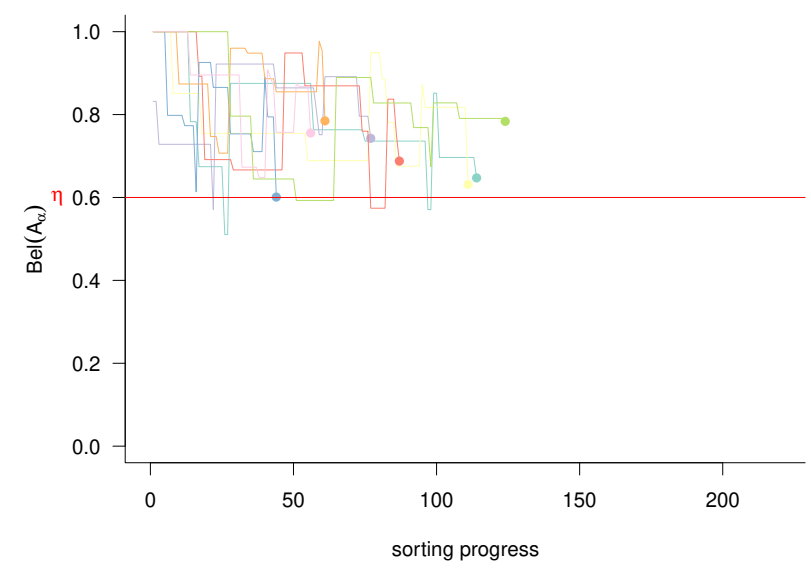

Fig. 6. Flexible procedure

4) Computational complexity: Let us denote $l$ the number of iterations carried out until the filling of the container. Obviously, $l \geq f$ inal, with equality when no fragment were rejected. In the case of the naive procedure, $A_{\alpha}$ is compared only to two intervals for each fragment $f$ to determine $\operatorname{Bel}\left(A_{\alpha} \mid f\right)$. Indeed, in this illustration we considered that $m(. \mid f)$ has only two focal elements. Thus, the complexity is linear in this case, i.e., computational complexity is $\mathcal{O}(l)$.

Concerning the approaches that take into account the updated evidence about the container composition, as in the cautious and flexible procedures, the number of focal elements associated to $m_{k}=m_{k-1} \oplus m\left(\mid f_{k}\right)$ grows exponentially related to the number of fragments $f_{k}$ in the deposit. Indeed, if $\mathcal{F}_{k}$ denotes the set of focal elements of $m\left(. \mid f_{k}\right)$, we have at most

$$
\left|\mathcal{F}_{1}\right| \times\left|\mathcal{F}_{2}\right| \ldots\left|\mathcal{F}_{k}\right|
$$

focal elements for $m_{k}$. In this illustration we considered that $\left|\mathcal{F}_{k}\right|=2$, for all fragments $f_{k}$. Thus, $m_{k}$ has at the most $2^{k}$ focal elements.

In the cautious procedure, it is necessary to determine $\operatorname{Bel}_{k}\left(A_{\alpha}\right)$ for all the $l$ fragments, including those rejected. The cost of these operations is lower than $\sum_{j=1}^{l} 2^{j}$. Thus the cautious procedure has an exponential complexity $\mathcal{O}\left(2^{l}\right)$.

The flexible procedure is more costly than the cautious one and its complexity is also exponential. Indeed, two focal elements for each mass $m^{\text {part } 1}$ and $m^{\text {part2 }}$ should be taken into account at each step. Thus $2^{k+2}$ total focal elements are considered and the computational complexity is $\mathcal{O}\left(2^{l}\right)$.

To reduce the number of focal elements, one may use approximation, for instance considering that "very close" focal elements as the same. For example, intervals $\mathcal{I}^{1}=\left[l^{1}, u^{1}\right]$ and
$\mathcal{I}^{2}=\left[l^{2}, u^{2}\right]$ may be seen as "very close" if the Euclidean distance between all intervals bounds of $\mathcal{I}^{1}$ and $\mathcal{I}^{2}$ is below a small positive real value $\epsilon$ :

$$
\sqrt{\sum_{i=1}^{n}\left[\left(l_{i}^{1}-l_{i}^{2}\right)^{2}+\left(u_{i}^{1}-u_{i}^{2}\right)^{2}\right]}<\epsilon .
$$

On the other hand, one may consider that focal elements to which a very small mass is assigned could be removed and their masses can be transferred to the whole set as ignorance.

\section{CONCLUSION}

This paper proposes tools to represent and make inference on the unit simplex in the framework of belief functions. This work focuses on summing proportions represented first by intervals then by mass function. In the case of mass functions, the paper proposed an easy way to make inference in order to obtain belief function and plausibility function of some specific events. This work is illustrated on the plastic sorting problem where the issue is to control the proportions of some plastic types in the sorting container. Three sorting procedures are proposed to deal with the problem of impurities constraints that the sorting container should respect. The flexible procedure is the most interesting one. As perspectives for this work, one should investigate more deeply the computation complexity to ensure the integration of the flexible procedures to a real-time sorting machine taking into account the industrial constraints. Also, we can extend this work to more general focal elements than convex ones.

\section{REFERENCES}

[1] G. Shafer, A mathematical theory of evidence. Princeton university press, 1976, vol. 42.

[2] P. Smets and R. Kennes, "The transferable belief model," Artificial intelligence, vol. 66, no. 2, pp. 191-234, 1994.

[3] A. P. Dempster, "Upper and lower probabilities induced by a multivalued mapping," in Classic Works of the Dempster-Shafer Theory of Belief Functions. Springer, 2008, pp. 57-72.

[4] N. Pellicanò, S. Le Hégarat-Mascle, and E. Aldea, "2cobel: A scalable belief function representation for $2 \mathrm{~d}$ discernment frames," International Journal of Approximate Reasoning, vol. 103, pp. 320-342, 2018.

[5] L. M. De Campos, J. F. Huete, and S. Moral, "Probability intervals: a tool for uncertain reasoning," International Journal of Uncertainty, Fuzziness and Knowledge-Based Systems, vol. 2, no. 02, pp. 167-196, 1994.

[6] J. Beigbeder, D. Perrin, J.-F. Mascaro, and J.-M. Lopez-Cuesta, "Study of the physico-chemical properties of recycled polymers from waste electrical and electronic equipment (weee) sorted by high resolution near infrared devices," Resources, Conservation and Recycling, vol. 78, pp. $105-114,2013$

[7] L. Jacquin, A. Imoussaten, F. Trousset, J. Montmain, and D. Perrin, "Evidential classification of incomplete data via imprecise relabelling: Application to plastic sorting," in International Conference on Scalable Uncertainty Management. Springer, 2019, pp. 122-135.

[8] C. Signoret, A.-S. Caro-Bretelle, J.-M. Lopez-Cuesta, P. Ienny, and D. Perrin, "Mir spectral characterization of plastic to enable discrimination in an industrial recycling context: Ii. specific case of polyolefins," Waste Management, vol. 98, pp. 160-172, 2019. 